\title{
Modification of $\mathrm{TiO}_{2}(001)$ surface electronic structure by $\mathrm{Au}$ impurity investigated with density functional theory
}

\author{
E. Mete, ${ }^{1, *}$ O. Gülseren, ${ }^{2}$ and Ş. Ellialtıoğlu ${ }^{3}$ \\ ${ }^{1}$ Department of Physics, Balıkesir University, Çă̆lş Campus, Balıkesir 10145, Turkey \\ ${ }^{2}$ Department of Physics, Bilkent University, Ankara 06800, Turkey \\ ${ }^{3}$ Department of Physics, Middle East Technical University, Ankara 06531, Turkey
}

(Received 27 February 2009; revised manuscript received 21 April 2009; published 17 July 2009)

\begin{abstract}
We have used density functional theory calculations based on the projector augmented wave method to investigate the electronic structure of Au-incorporated anatase $\mathrm{TiO}_{2}(001)$ surface. Due to the coordination with several level oxygens, $\mathrm{Au}$ atoms can be encapsulated inside $\mathrm{TiO}_{2}$ slab. Au is adsorbed over the surface Ti-O bond, so-called the bridge site on anatase $\mathrm{TiO}_{2}(001)-1 \times 1$ surface. However, for 0.25 monolayer coverage, $\mathrm{Au}$ atoms energetically prefer to stay at $0.64 \AA$ above the midpoint of the two surface oxygens which is significantly closer to the surface layer. When implanted inside the slab for full coverage, Au forms parallel metallic wires inside $\mathrm{TiO}_{2}$ lattice where interlayer distances increase due to local segregation. Au brings half-filled impurity states into the band gap leading to metallization, in addition to other filled surface and impurity bands within the gap. These Au-driven Fermi-level-pinning gap states are close to, or even in some cases inside, the conduction band of the host slab. On the other hand, if Au is substituted for the surface Ti atom, Fermi level falls lower in the gap closer to the valence-band top.
\end{abstract}

DOI: $10.1103 /$ PhysRevB.80.035422

PACS number(s): 73.20.Hb, 68.43.Bc

\section{INTRODUCTION}

Lately, titania $\left(\mathrm{TiO}_{2}\right)$ has received an increased amount of attention since it is considered to be promising in costeffective photovoltaic applications. However, high reactivity of $\mathrm{TiO}_{2}$ only under UV light bears a great disadvantage which reduces the quantum efficiency so that pure $\mathrm{TiO}_{2}$ is not sufficient alone for a practical system application. ${ }^{1-5}$

McFarland and Tang, ${ }^{6}$ in a recent work, proposed an $\mathrm{Au} / \mathrm{TiO}_{2} / \mathrm{Ti}$ multilayer photovoltaic device on which photon absorption occurs in the deposited dye molecules while electron-hole pairs are created upon light illumination inside the semiconductor for the conventional solid-state solar cells. This ultrathin metal-semiconductor-junction Schottky diode has driven particular attention to gold-titania interface. ${ }^{7}$

Gold has been proposed to enhance the catalytic activity of anatase $\mathrm{TiO}_{2} \cdot{ }^{8,9}$ Au-incorporated anatase-based nanocatalysts have been synthesized for device applications. ${ }^{10-12}$ Moreover, diffusion of gold into anatase polymorph of titania has also been reported. ${ }^{13}$

The anatase phase of titanium dioxide, although being less stable than the rutile polymorph, is catalytically more active. ${ }^{14-16}$ In this sense, the surface properties are of major importance. There are several studies on the topological and electronic structure of the single crystals of anatase $\mathrm{TiO}_{2}(001)$ surface. ${ }^{17-20}$ In this paper, we investigated the reconstructive effect of various types of $\mathrm{Au}$ incorporation in the anatase substrate and the role of such an impurity on the electronic structure of $\mathrm{TiO}_{2}(001)$. We considered $\mathrm{Au}$ in and on the surface at quarter and full monolayer (ML) concentrations including $\mathrm{Au}(\mathrm{Ti})$ substitutional cases as well as gold dimer adsorption on the surface. We have discussed their thermodynamic stabilities for various experimental environments.

\section{METHOD}

The total-energy density functional theory (DFT) calculations have been performed within the generalized gradient approximation for the exchange-correlation effects via Perdew-Burke-Ernzerhof functional, ${ }^{21}$ using plane-wave basis sets and the projector augmented waves method ${ }^{22,23}$ as implemented in the Vienna $a b$ initio simulation package. ${ }^{24}$

Anatase $\mathrm{TiO}_{2}(001)$ stoichiometric surface has been modeled as a symmetrical slab which constitutes six $\mathrm{TiO}_{2}$ layers in a supercell with a vacuum region of at least $13 \AA . \mathrm{A} \mathrm{TiO}_{2}$ layer consists of three atomic planes in which bridging oxygen atoms are out of the level Ti plane. Our choice regarding the number of layers represent one of the thickest slab models among the other theoretical studies. ${ }^{17-20}$ Moreover, for $\mathrm{Au}$ implantation deeper than the fourth atomic plane we used 8- $\mathrm{TiO}_{2}$-layer slab model to avoid any interaction between the $\mathrm{Au}$ impurities within the same supercell which are implanted from the bottom and from the top surfaces.

For the gold-incorporated surface models we kept ions, only in the central $\mathrm{TiO}_{2}$ layer, fixed to their bulk positions because the forces on these remain to be insignificantly small in all of the calculations. We employed a conjugate-gradients algorithm to compute the electronic ground state based on the minimization of the total-energy subject to a convergence tolerance of $0.1 \mathrm{meV}$. Geometry optimization has been performed, subsequently, by the reduction in the quantum force on each of the unconstrained ions to less than $10 \mathrm{meV} / \AA$.

Single-particle wave functions have been expanded in terms of plane waves up to a cutoff-energy value of $400 \mathrm{eV}$. The Brillouin-zone integrations have been carried out over 32 and five special $k$ points sampled in the irreducible wedge for $1 \times 1$ and $2 \times 2$ surfaces, respectively. We did not impose any symmetry in our surface calculations.

In thermodynamic equilibrium, the most stable surface composition that is entirely surrounded by a thermal bath at 
temperature $T$ under a given pressure $p$ minimizes the surface Gibbs free energy (GFE) $\gamma(T, p) .{ }^{25-28}$ Therefore, relative stability of the phases can be expressed in terms of the difference $\Delta \gamma(T, p)$ in the GFE of impure surface structures and relaxed clean surface as

$$
\begin{aligned}
\Delta \gamma(T, p)= & \frac{1}{A}\left\{G_{{\mathrm{Au} / \mathrm{TiO}_{2}}_{2}}\left(T, p, \Delta n_{\mathrm{Ti}}, \Delta n_{\mathrm{Au}}\right)-G_{\mathrm{TiO}_{2}}(T, p)\right. \\
& \left.-\Delta n_{\mathrm{Ti}} \mu_{\mathrm{Ti}}(T, p)-\Delta n_{\mathrm{Au}} \mu_{\mathrm{Au}}(T, p)\right\},
\end{aligned}
$$

where $G_{\mathrm{Au} / \mathrm{TiO}}$ and $G_{\mathrm{TiO}_{2}}$ are the GFEs of the Au-incorporated and reference clean $\mathrm{TiO}_{2}$ surface slabs, respectively. $A$ is the corresponding unit-cell area also functioning as normalization for different stoichiometries. As the name suggests, $\Delta n$ is the difference in the number of atoms from that of the reference surface, while $\mu$ denotes the chemical potential for the referring species. A negative $\Delta \gamma$ value indicates a more stable structure relative to the clean $\mathrm{TiO}_{2}$ surface. Positive values, on the other hand, give the relative formation energy that is required to assemble the corresponding composition.

$\mathrm{GFE}$, in general, is defined by $G=U+p V-T S$. One can omit the $p V$ term in comparison to the surface energy for the pressures, $p$, under consideration as well as the $T S$ term since the contribution from the entropy, $S$, is negligible. Therefore, it can be approximately expressed only by the internal energy $U$. If one also neglects the ionic vibrational contributions, $U$ will be equal to the total energy $E_{\text {total }}(N, V)$ obtained by the DFT slab calculations.

Thermodynamic equilibrium is established when the chemical potential of a given atomic species becomes equal in all phases that come into contact with each other. In particular, we assume that the surface structures are in equilibrium with the anatase $\mathrm{TiO}_{2}$ bulk so that $\mu_{\mathrm{Ti}}+2 \mu_{\mathrm{O}}=\mu_{\mathrm{TiO}}$. Moreover, the reference energies at the most stable elemental phases of $\mathrm{Ti}$ and $\mathrm{Au}$ set the upper bounds for their corresponding chemical potentials. For instance, the maximum value of $\mu_{\mathrm{Ti}}$ can be accessed in the hcp Ti bulk solid phase. Otherwise, the bulk phase would be unstable with respect to precipitation of bulk Ti. Similarly, $\mu_{\mathrm{Au}}$ cannot be above the chemical potential of ccp gold bulk. Molecular oxygen defines the upper boundary for $\mu_{\mathrm{O}}$ so that $\mu_{\mathrm{O}}=\frac{1}{2} E_{\mathrm{O}_{2}}$ which corresponds to an O-rich experimental environment, therefore, defining the minimum value of $\mu_{\mathrm{Ti}}$ through the thermodynamic equilibrium condition $\mu_{\mathrm{Ti}}+2 \mu_{\mathrm{O}}=\mu_{\mathrm{TiO}_{2}}$.

\section{RESULTS AND DISCUSSION}

We systematically studied Au impurities on and inside the anatase $\mathrm{TiO}_{2}(001)$ stoichiometric surface. The role of such an incorporation on the electronic properties of anatase surface has been investigated by examining adsorptional, substitutional, and, interstitial impurities at quarter and full coverages. For these concentrations, we considered gold ions in the subsurface at the interstitial cavities up to a depth of the fifth atomic plane and also substituted them for Ti ions up to the third Ti plane.

The topological structure and the electronic properties of the bare anatase $\mathrm{TiO}_{2}(001)$ has been discussed in detail
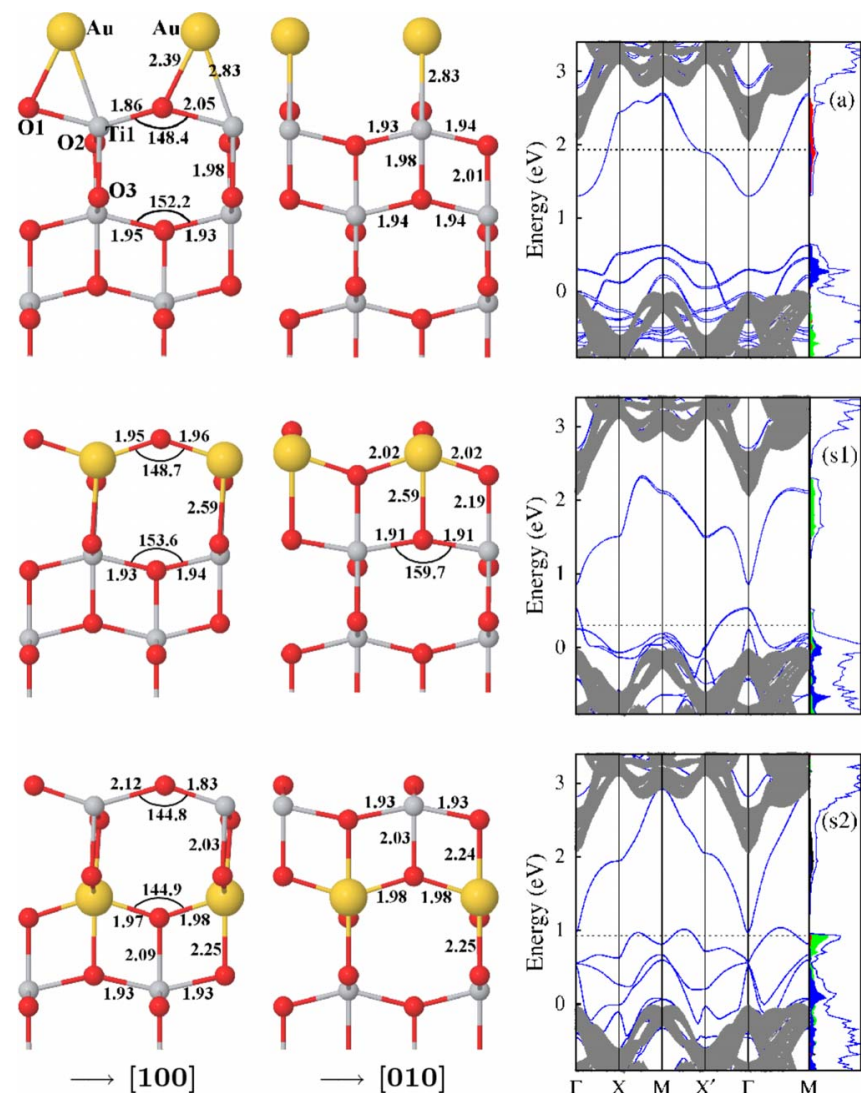

FIG. 1. (Color online) The geometric and electronic structures of a- $1 \times 1$ (first row), s1-1 $1 \times 1$ (second row), and s2-1 $\times 1$ (last row) for the $\mathrm{Au} / \mathrm{TiO}_{2}(001)-1 \times 1$ systems. Big light gray (yellow) balls represent gold. Small dark (red) and bright (gray) balls denote oxygen and titanium atoms, respectively. Bond angles and bond lengths are given in degrees and angstroms, respectively. Energy bands and DOS structures are described in detail in the corresponding subsections.

elsewhere. ${ }^{29}$ Figure 1 shows the optimized geometries and corresponding electronic structures of $\mathrm{Au}-\mathrm{TiO}_{2}$ combined systems for $1 \times 1$ surface. The first two panels to the left show the side views of the geometric structures along [100] and [010] directions. In addition, relevant electronic structures are presented next to these geometries at each row representing a distinct impurity case. Each ion is labeled with respect to the atomic plane that it belongs to for each of species separately. For instance, an oxygen at the second oxygen atomic layer is labeled as $\mathrm{O} 2$. Furthermore, bulk termination gives rise to undercoordinated ions by breaking the axial bonds over the surface. As a result, $\mathrm{O} 1$ and Til become twofold and fivefold coordinated, thus, also referred as $\mathrm{O} 2 \mathrm{c}$ and Ti5c, respectively.

\section{A. $\mathrm{Au} / \mathrm{TiO}_{2}(001)-(1 \times 1)$}

\section{Adsorptional case: $a-1 \times 1$}

Au-adsorptional case, that the first row of Fig. 1 refers to, has been obtained by relaxing all possible initial configurations in which $\mathrm{Au}$ comes into contact with the active sites on the support surface. Truly, in this resultant geometry, Au re- 
TABLE I. Calculated electronic and structural parameters for $\mathrm{Au}-\mathrm{TiO}_{2}(001)$ anatase system: work function, position of the Fermi energy relative to bulk valence-band maximum, Au depth relative to surface oxygens, and $\mathrm{Au}-\mathrm{O}$ and $\mathrm{Au}-\mathrm{Ti}$ distances for each model labeled in compliance with Figs. 1-3.

\begin{tabular}{cccccc}
\hline \hline Model & $\begin{array}{c}W \\
(\mathrm{eV})\end{array}$ & $\begin{array}{c}E_{\mathrm{F}} \\
(\mathrm{eV})\end{array}$ & $\begin{array}{c}h_{\mathrm{Au}} \\
\AA\end{array}$ & $\begin{array}{c}d_{\mathrm{Au}-\mathrm{O}} \\
\AA\end{array}$ & $\begin{array}{c}d_{\mathrm{Au}-\mathrm{Ti}} \\
\AA\end{array}$ \\
\hline $\mathrm{a}-1 \times 1$ & 5.45 & 1.93 & 2.14 & $2.39(\mathrm{O} 1)$ & $2.83(\mathrm{Ti} 1)$ \\
$\mathrm{s} 1-1 \times 1$ & 6.37 & 0.31 & -0.51 & $1.95(\mathrm{O} 1), 2.03(\mathrm{O} 2)$ & $3.51(\mathrm{Ti} 2)$ \\
$\mathrm{s} 2-1 \times 1$ & 6.06 & 0.93 & -3.22 & $2.24(\mathrm{O} 2), 1.98(\mathrm{O} 3, \mathrm{O} 4), 2.25(\mathrm{O} 5)$ & $3.23(\mathrm{Ti} 1), 3.28(\mathrm{Ti} 3)$ \\
$\mathrm{a}-2 \times 2$ & 5.02 & 2.02 & 1.94 & $2.20(\mathrm{O} 1)$ & $2.68(\mathrm{Ti} 1)$ \\
$\mathrm{b}-2 \times 2$ & 4.29 & 2.60 & 0.64 & $2.06(\mathrm{O} 1), 2.73(\mathrm{O} 2)$ & $3.06(\mathrm{Ti} 1)$ \\
$\mathrm{c}-2 \times 2$ & 4.33 & 2.59 & -2.71 & $3.17(\mathrm{O} 2), 2.09(\mathrm{O} 3), 2.50(\mathrm{O} 4)$ & $2.85(\mathrm{Ti} 1), 2.77(\mathrm{Ti} 2)$ \\
$\mathrm{d}-2 \times 2$ & 4.49 & 2.57 & -3.40 & $2.46(\mathrm{O} 3), 2.05(\mathrm{O} 4), 3.13(\mathrm{O} 5), 2.74(\mathrm{O} 6)$ & $2.80(\mathrm{Ti} 2), 2.94(\mathrm{Ti} 3)$ \\
$\mathrm{e}-2 \times 2$ & 4.47 & 2.58 & -5.24 & $3.11(\mathrm{O} 4), 2.06(\mathrm{O} 5), 2.49(\mathrm{O} 6), 3.55(\mathrm{O} 7)$ & $2.93(\mathrm{Ti}), 2.78(\mathrm{Ti} 3)$ \\
$\mathrm{s} 1-2 \times 2$ & 6.80 & 0.31 & -0.27 & $2.01(\mathrm{O} 1), 2.03(\mathrm{O} 2)$ & $3.37(\mathrm{Ti} 2)$ \\
$\mathrm{s} 2-2 \times 2$ & 6.66 & 0.58 & -3.18 & $2.07(\mathrm{O} 2), 2.59(\mathrm{O} 3), 2.02(\mathrm{O} 4), 2.03(\mathrm{O} 5)$ & $3.45(\mathrm{Ti} 1), 3.18(\mathrm{Ti} 3)$ \\
$\mathrm{aa}-2 \times 2$ & 6.28 & 1.04 & 1.57 & $2.32(\mathrm{Au} 1-\mathrm{O} 1), 2.07(\mathrm{Au} 2-\mathrm{O} 1)$ & $2.66(\mathrm{Au} 1-\mathrm{Ti} 1), 2.67(\mathrm{Au} 2-\mathrm{Ti} 1)$ \\
\hline \hline
\end{tabular}

laxes to a bridge position on $\mathrm{O} 1$ and Ti1 that reflects the maximum interaction between the $\mathrm{Au}$ adsorbate and the surface. At this minimum-energy position, O1-Au-Ti1 angle becomes $45.4^{\circ}$ with $\mathrm{Au}-\mathrm{O} 1$ and Au-Ti1 bond distances being 2.39 and $2.83 \AA$, respectively. The reconstructive effect of the adsorbate on the lattice remains negligibly small at the subsurface layers. Au adsorption at $1 \mathrm{ML}$ coverage adapts an ideal-like $\mathrm{TiO}_{2}$ atomic arrangement by saturating the dangling bonds. Yet, the symmetry breaking in the Ti1-O1 bonds which stems from the relaxation of the clean surface is still not lifted as bond lengths read 2.05 and $1.86 \AA$. The structural parameters as well as some other physical entities related to electronic properties such as the surface work function and Fermi energy relative to bulk valence-band maximum (VBM) are presented in Table I.

$\mathrm{Au}$ adsorbate brings a two-dimensional impurity state in the gap closer to the conduction band (CB). Fermi level passes through the saddle point at $\mathrm{X}^{\prime}$ of this band corresponding to a logarithmic singularity in the site-projected local density of states (LDOS). This half-filled Fermi-levelpinning state, therefore, leads to metallization. The reactivity of the $\mathrm{O} 1$ site is higher than that of Ti1, which is also apparent from the LDOS presented on the rightmost panel of the corresponding electronic band structure in Fig. 1. A group of four occupied defect states lie distinctly below the Fermi energy derived from the valence band (VB) possessing dominant $\mathrm{O} 1$ character. The contribution of Ti1 to the total DOS around the Fermi energy is very weak.

\section{Substitutional case: $\mathrm{s} 1-1 \times 1$}

The most noticeable effect of Au substitution for surface Ti (Ti1) at $1 \times 1$ structure, also referred as s $1-1 \times 1$, is that the distance between the top and the second $\mathrm{TiO}_{2}$ layer increases substantially. For instance, the Ti1-O3 axial bond length of $1.96 \AA$ at the clean surface extends to $2.59 \AA$ for $\mathrm{Au}-\mathrm{O} 3$. In addition to the increase in the interlayer distance, this extension also gets the Ti2-O3-Ti2 angle to enlarge from $152.0^{\circ}$ to $159.7^{\circ}$ at the second $\mathrm{TiO}_{2}$ layer. Moreover, Au-O1 equatorial bonds along [100] at the surface layer become almost equal being 1.95 and $1.96 \AA$ as shown in the second row of Fig. 1.

Unlike that of the adsorptional case, Au-Til substitution drives Fermi energy to fall lower in the gap closer to the valence-band top. Because of the partially occupied nature of this state which is populated by $\mathrm{Au}, \mathrm{O} 1$, and chiefly by $\mathrm{O} 2$, the combined system goes into the metallic regime. The upper lying unoccupied defect state, which takes up a big portion of the gap to the energies above the Fermi level as a result of the strong dispersion, shows dominant $\mathrm{Au}$ and $\mathrm{O} 2$ contribution.

\section{Substitutional case: $\mathrm{s} 2-1 \times 1$}

The optimized geometry presented in Fig. 1 (s2) for the second substitutional case $(\mathrm{s} 2-1 \times 1)$ has been obtained by relaxing an initial configuration which was formed by replacing $\mathrm{Ti} 2$ with $\mathrm{Au}$ in the bare $8-\mathrm{TiO}_{2}(001)$-layer slab model. $\mathrm{Au}$ impurity sits below the surface in the second $\mathrm{TiO}_{2}$ layer having extended interlayer distances, similar to s1-1 $\times 1$ case, of 2.24 and $2.25 \AA$ with the top and the third layers, respectively. On the other hand, Ti1-O1 bond lengths remain to be nonequivalent with values of 2.12 and $1.83 \AA$ making a Ti1-O1-Ti1 angle of $144.8^{\circ}$.

Subsurface substitutional gold sets the Fermi level at 0.93 $\mathrm{eV}$ above the VBM pinned by a strongly dispersed empty defect state, of width $\sim 2 \mathrm{eV}$, which descends from the CB. The second band below, with a width of about $0.5 \mathrm{eV}$, is partially filled causing metallization of the system. This band is degenerate with a third band of similar width at $\Gamma$ point and all these three states are symmetrical with respect to $M$ point along $\overline{\Gamma X M X^{\prime} \Gamma}$. The unoccupied first band is originating mainly from the $\mathrm{Au}-\mathrm{O} 4$ and $\mathrm{Au}-\mathrm{Au}$ interactions. The half-occupied second band shows dominant $\mathrm{O} 2$ and relatively weaker $\mathrm{O} 4$ character. Third band has an LDOS peak stemming from $\mathrm{O} 3$. The fourth band in the gap which crosses the third along most of $\overline{\mathrm{XMX}^{\prime}}$ is due to the clean surface [see Fig. 2(a) in Ref. 18]. The LDOS for the surface oxygen (O1) 

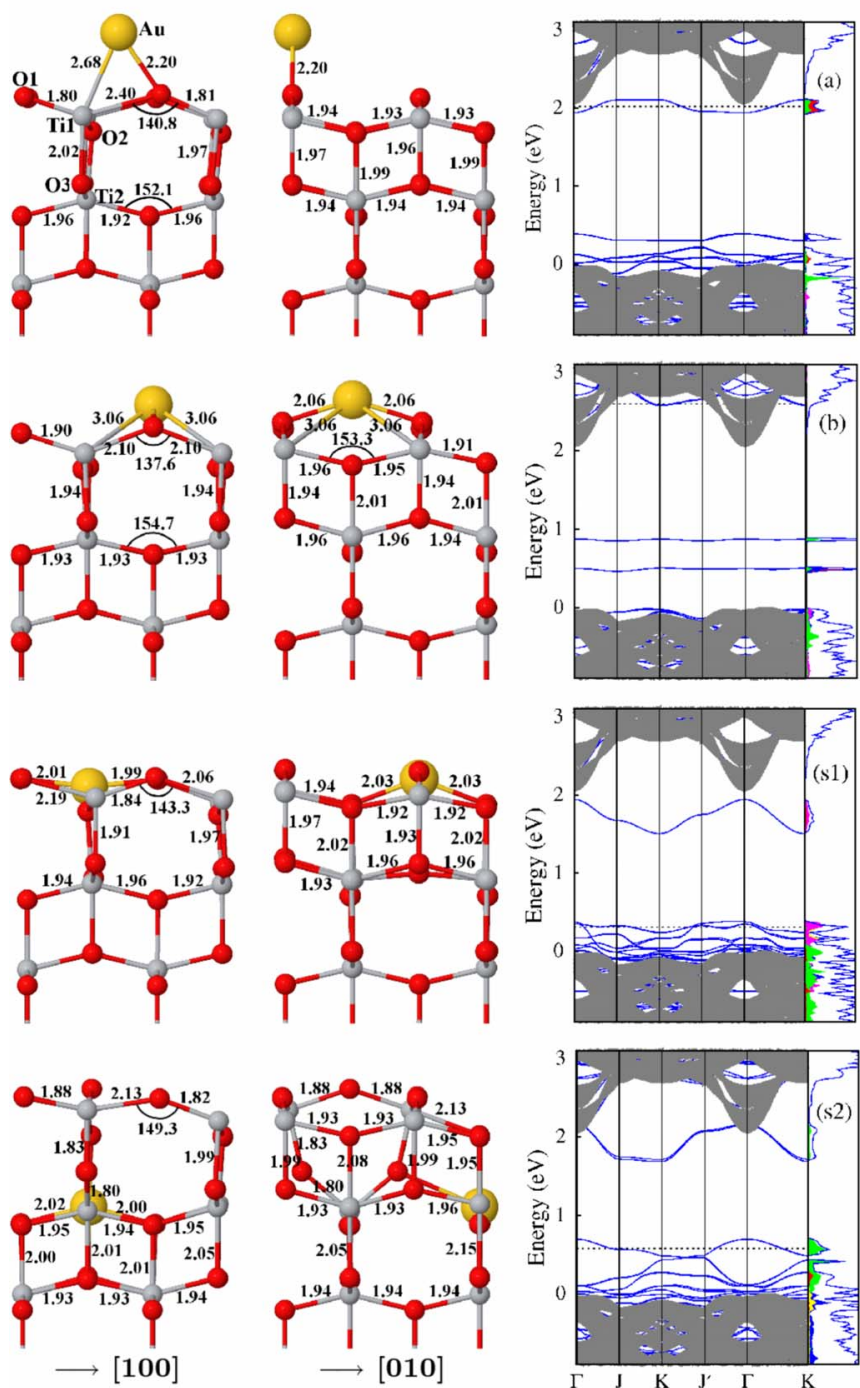
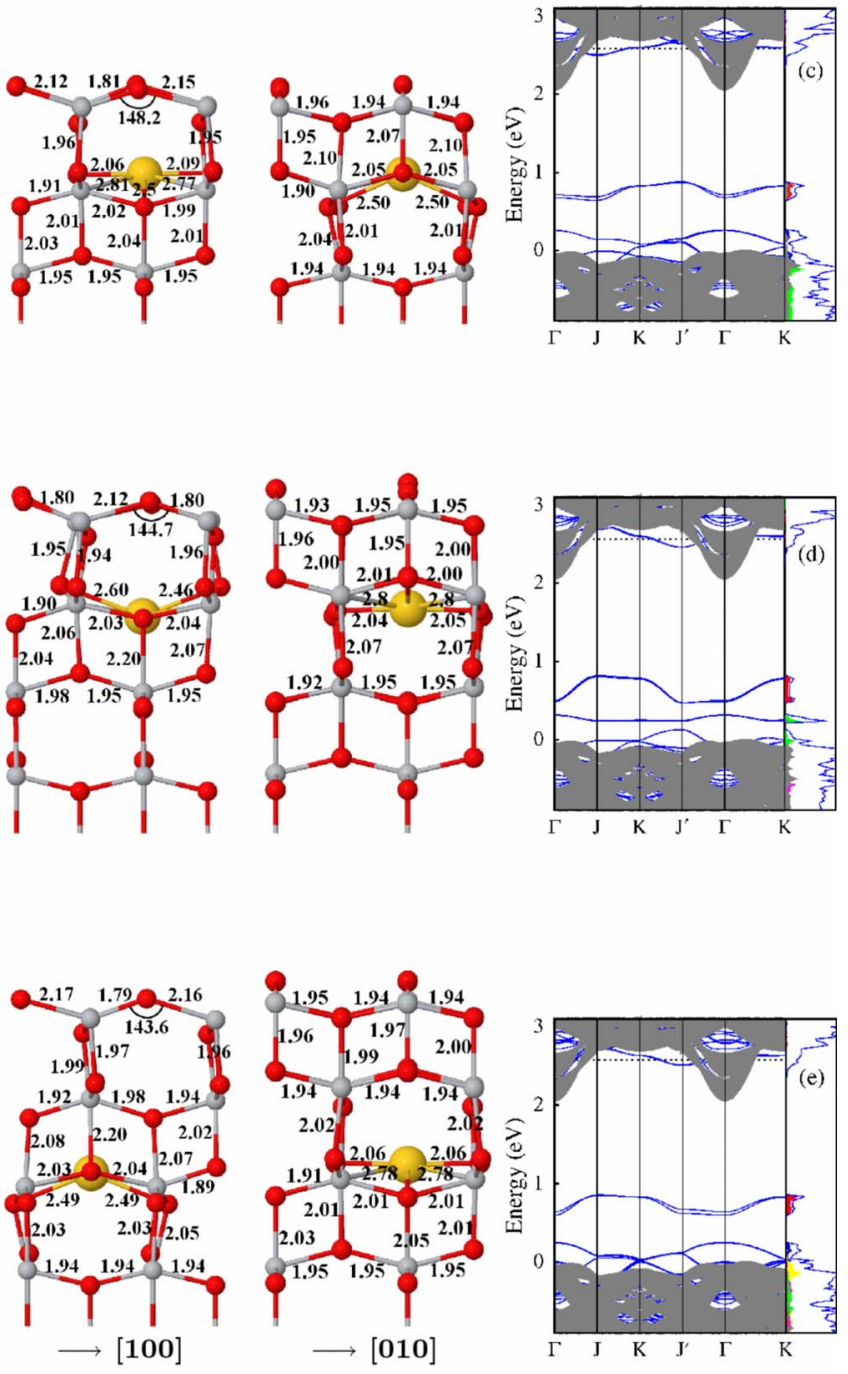

FIG. 2. (Color online) Geometric and electronic structures of $\mathrm{Au} / \mathrm{TiO}_{2}(001)-2 \times 2$ systems for the adsorptional, substitutional, and interstitial gold incorporations. Label for each case, is given in the DOS panel, should read as, e.g., a- $2 \times 2$ for the first row, and so on. Naming convention for the atoms follow those of Fig. 1. The bond lengths and angles are given in angstroms and in degrees, respectively.

disperses over $\mathrm{VB}$ similar to that of $\mathrm{s} 1-1 \times 1$ implying a charge transfer to subsurface cation sites. This results in a work function of $6.06 \mathrm{eV}$. We have also considered Au substitution for Ti3 (not shown). The band structure for s3-1 $\times 1$ has common characteristics with that of $s 2-1 \times 1$. The first and second bands are very similar, third is no longer degenerate with the second at $\Gamma$, and fourth, the surface state, is pushed down toward the VB and is also symmetric with respect to $M$. In quest of finding a trend we checked the band structures for Au substitution even deeper, namely, for Ti5 $(\mathrm{s} 5-1 \times 1)$ and also considered a case for bulk substitution. Comparison of these series of cases, Au for Ti1 to bulk Ti, band structures projected to the same surface Brillouin zone, shows that the second and third bands continue to repel each other, while $E_{F}$ passes through the second defect band as before.

\section{Interstitials at $1 \times 1$}

For the interstitial case at $1 \times 1$ surface, strong metalmetal interaction distorts the local lattice structure drastically due to shortened Au-Au distance of $3.76 \AA$. This causes a local segregation that leads to the formation of parallel metallic $\mathrm{Au}$ wires along [010] inside $\mathrm{TiO}_{2}$ lattice where interlayer distances further increase (from $\sim 2.02$ to $3.89 \AA$ ).

$$
\text { B. } \mathrm{Au} / \mathrm{TiO}_{2}(001)-(2 \times 2)
$$

\section{Adsorptional case: a-2 $2 \times 2$}

Similar to the case for the $1 \mathrm{ML}$ coverage, single $\mathrm{Au}$ adsorbate at the $2 \times 2$ surface is twofold coordinated with $\mathrm{O} 1$ and Ti1 at the bridge position over the Ti1-O1 bond as shown in Fig. 2(a). Au promotes the nearest-neighbor Ti1 upward elongating the Ti1-O3 bond from a clean surface value of 1.96 to $2.02 \AA$. It also interacts strongly with the nearest O1 which is elevated up leading to a significant increase in the Ti1-O1 bondlength, from 2.16 to $2.40 \AA$, over which $\mathrm{Au}$ stays at the minimum-energy position. As a result, O1Ti1-O1 angle gets wider by $4^{\circ}$ compared to the value at the clean surface. Quarter ML Au adsorption yields significant 
differences in the atomic positions from those of the $1 \mathrm{ML}$ coverage. For instance, Au-Ti1 and Au-O1 bond lengths become shorter (2.68 and $2.20 \AA)$ than the values $(2.83$ and $2.39 \AA$ ) for 1 ML coverage, respectively. This can be explained by the increased charge transfer between the impurity and the support surface due to much weaker Au-Au impurity interaction at $0.25 \mathrm{ML}$ coverage.

For a- $2 \times 2$ case, energy-gap region is characterized by weakly dispersing six defect states as shown in Fig. 2(a). The one lying just below the $\mathrm{CB}$ that is half filled by simple electron counting drives the combined system into metallic state. This Au-O1 driven state is flat along $\overline{\mathrm{JK}}$ and almost flat along $\overline{J^{\prime} \Gamma}$ and therefore quasi-one dimensional (quasi-1D) in nature as seen also in LDOS. The next defect state that lies $1.56 \mathrm{eV}$ below the Fermi energy at $\Gamma$ also being quite flat, especially along $\overline{\mathrm{JKJ}^{\prime}}$, has mainly $\mathrm{O} 2$ character. The remaining four states come up slightly above the VBM with many crossings originating mainly from $\mathrm{Au}-\mathrm{O} 1$ and $\mathrm{Au}-\mathrm{Ti1}$ interactions.

\section{Adsorptional case: $\mathrm{b}-2 \times 2$}

In the second adsorptional model, namely, b- $2 \times 2, \mathrm{Au}$ relaxes into a very symmetrical position $0.64 \AA$ above the midpoint between two $\mathrm{O} 1$ ions. It forms two and four equidistant bonds with those $\mathrm{O} 1$ ions and with Ti1s that read $2.06 \AA$ for the former and $3.06 \AA$ for the latter, respectively. This isotropic coordination with two nearest-neighbor O1s and with four surface Ti's get Ti1-O3 bonds to be aligned parallel to [001]. Hence, each of the Ti1-O1 bonds become equal to $2.10 \AA$ in length making an Ti1-O1-Ti1 angle of $137.6^{\circ}$. On the other hand, two Ti2-O2 bonds that are coplanar with $\mathrm{Au}$ remain to be slightly skewed with congruent angles of $3.6^{\circ}$ each.

Fermi level falls into the $\mathrm{CB}$ by a resonant defect state causing metallization for the electronic structure presented in Fig. 2(b). Two flat going occupied defect states lie about 0.5 and $0.9 \mathrm{eV}$ above VBM. The higher one of these bands carries dominant $\mathrm{O} 1$ character, whereas the lower one originates from $\mathrm{Au}-\mathrm{O} 1$ interaction with larger $\mathrm{Au}$ mixing and has some $\mathrm{O} 2$ contribution as well.

\section{Substitutional case: $\mathrm{s} 1-2 \times 2$}

When substituted for surface titanium as shown in Fig. 2 (s1), Au relaxes into a position $0.38 \AA$ above the original Ti1 lattice point where it forms two Au-O1 bonds with slightly unequal lengths of 1.99 and $2.01 \AA$. It is also twofold coordinated to two nearest-neighbor $\mathrm{O} 2 \mathrm{~s}$ at a distance of $2.03 \AA$ away from each of them. The inequivalency in the Ti-O1 bond lengths still remains, being 1.84 and $2.06 \AA$, over the undercoordinated surface oxygen row along [100] next to the row in which $\mathrm{Au}$ is substituted. Moreover, Au-O3 separation elongates to $2.65 \AA$ which can be attributed to $\mathrm{Au}$ attaining a nominal charge state that weakens the charge transfer from $\mathrm{Au}$ to O3. Resulting bond length of Ti2 with this $\mathrm{O} 3$ shortens to $1.85 \AA$ while the other Ti2-O3 distances are all $1.96 \AA$.

A stack of surface bands disperse over an energy range of $0.33 \mathrm{eV}$ with intercrossings just above VBM. The highest of those is half occupied, setting the Fermi energy at $0.31 \mathrm{eV}$ relative to bulk valence-band top. Therefore, combined system has been predicted to show metallic behavior. The two sharp LDOS peaks at and just below the Fermi level in Fig. 2 (s1) are mainly due to $\mathrm{O} 1$ which is bonded to Au. The rather lower part of those surface states, on the other hand, originate from undercoordinated $\mathrm{O} 1 \mathrm{~s}$ which are away from $\mathrm{Au}$ site. An empty defect state falls into the gap region at around $2 \mathrm{eV}$ at $\Gamma$ below $\mathrm{CB}$ having a minimum at $\mathrm{K}$. This band has two saddle points at $\mathrm{J}$ and $\mathrm{J}^{\prime}$ which are not at the same energy because of the unequal Au-O1 bondlengths along [100]. Corresponding LDOS peaks come out as a result of excess antibonding charge localized around gold.

\section{Substitutional case: $\mathrm{s} 2-2 \times 2$}

$\mathrm{Au}$ substitution for Ti2 causes local disturbances on the nearby $\mathrm{O} 3 \mathrm{~s}$ as a result of the excess charge embedded by and localized around the impurity site. Equatorial $\mathrm{Au}-\mathrm{O} 3$ bonds on (100) plane extend to $2.59 \AA$ leading to a significant dislocation of $\mathrm{O} 3 \mathrm{~s}$ from their lattice positions. This subsurface reconstruction induces stress on Ti1-O3 interaction that flips up Ti1-O2 bonds. Then, the displaced $\mathrm{O} 2$ levels itself with $\mathrm{O} 1$ atomic plane as shown in Fig. 2 (s2). On the other hand, subtle atomic rearrangements have been observed upon Au substitution for Ti2 over the planes perpendicular to [010] direction. In this structure, Ti1-O1 bonds are still unequal (2.13 and $1.82 \AA)$ making a Ti1-O1-Ti1 angle of $149.3^{\circ}$. Au substitution for a third-layer Ti cation, namely, s3-2 $2 \times 2$ dislocates the nearby $\mathrm{O} 3 \mathrm{~s}$ in the same way as $\mathrm{s} 2-2 \times 2$ does. This suggests that $\mathrm{Au}-\mathrm{Ti}$ replacement for deeper lying cations will cause similar local reconstruction.

The electronic structure of s2-2 2 model in Fig. 2 (s2) have similar characteristics with that of $s 1-2 \times 2$. Both of them have an unoccupied defect state arising chiefly from $\mathrm{Au}$ impurity. However, the one for the s2 case lies higher and partly inside $\mathrm{CB}$ at around $\Gamma$. It is slightly dispersed along $\overline{\mathrm{JK}}$ and $\overline{J^{\prime} \Gamma}$ causing the band to be a quasi-1D band as opposed to the quasi-two-dimensional band of s1 case. This also shows itself in their LDOS structures. An O2-driven halffilled defect state having a maximum at $\Gamma$ has been hived off from the lower lying group of surfacelike bands. Therefore, Fermi level is at $0.58 \mathrm{eV}$ with respect to bulk valence-band top. This is related to the surface oxygen, $\mathrm{O} 2$, that is bonded to $\mathrm{Au}$. The next band down is also of $\mathrm{O} 2$ character, however, it gets additional contributions from the surface oxygen, O2, raised to the level of $\mathrm{O} 1 \mathrm{~s}$, as well. In comparison with those of s1-2 $\times 2$, the stack of surface bands disperse over a relatively higher energy range of $0.47 \mathrm{eV}$ just above VBM. They are mainly due to axial $\mathrm{Au}-\mathrm{O} 2$ interaction. LDOS also shows contributions to bands slightly below VBM primarily from surface oxygens. As in the $1 \times 1$ case, we have examined the deeper Au substitutions for up to bulk Ti which lead the half-filled defect state to move about $1 \mathrm{eV}$ upward closer to the empty band in the gap.

\section{Interstitials at $2 \times 2$}

Au can be encapsulated at the interstitial cavities in the subsurface layers starting from the fourth atomic layer for $2 \times 2$ surface as opposed to $1 \times 1$. The first three possible 
TABLE II. Bader charge analysis of $\mathrm{Au}-\mathrm{TiO}_{2}(001)$ anatase systems. Atom labels follow Figs. 1-3. The values represent the valence charge states for lattice atoms that are closest to Au impurity site.

\begin{tabular}{ccccccc}
\hline \hline Model & O1 & O2 & O3 & Ti1 & Ti2 & Au \\
\hline Clean & -1.26 & -1.34 & -1.33 & +2.61 & +2.64 & \\
a-1 $\times 1$ & -1.20 & -1.34 & -1.33 & +2.58 & +2.64 & -0.03 \\
s1-1 $\times 1$ & -0.70 & -0.85 & -1.18 & & +2.64 & +1.38 \\
s2-1 $\times 1$ & -1.24 & -1.17 & -0.94 & +2.58 & & +1.54 \\
a-2 $\times 2$ & -1.15 & -1.34 & -1.32 & +2.55 & +2.65 & -0.04 \\
b-2 $\times 2$ & -1.18 & -1.35 & -1.33 & +2.57 & +2.62 & +0.34 \\
c-2 $\times 2$ & -1.26 & -1.33 & -1.24 & +2.55 & +2.56 & +0.34 \\
d-2 $\times 2$ & -1.25 & -1.34 & -1.30 & +2.60 & +2.56 & +0.34 \\
e-2 $\times 2$ & -1.26 & -1.34 & -1.34 & +2.60 & +2.58 & +0.38 \\
s1-2 2 & -0.95 & -1.11 & -1.26 & & +2.63 & +1.39 \\
s2-2 $\times 2$ & -1.20 & -1.03 & -1.27 & +2.63 & & +1.32 \\
aa-2 2 (Au1) & -1.23 & -1.35 & -1.32 & +2.56 & +2.64 & -0.07 \\
aa-2 $\times 2$ (Au2) & -1.12 & -1.35 & -1.33 & +2.54 & +2.64 & +0.05 \\
\hline \hline
\end{tabular}

structures are presented in Figs. 2(c)-2(e). As is common to all of them, Au relaxes into the midpoint between the twolevel oxygens where it also establishes equidistant coordination with four nearest-neighbor Ti's that lie at the closest $\mathrm{Ti}$ atomic plane. Au interstitial causes local disturbances such that two second nearest-neighbor oxygens at the preceding or succeeding $\mathrm{O}$ layers get slightly repelled out from their bulk lattice positions due to the induced stress incorporated by the excess charge at the impurity site. Au implantation to the internal cavities cannot help in lifting the symmetry breaking in the Ti1-O1 bond distances that exists in the case of the bare anatase surface.

The interstitial Au impurities in $\mathrm{TiO}_{2}(001)$ surface, corresponding to Figs. 2(c)-2(e), show similar electronic characteristics since they represent equivalent local environments. For all of them, Fermi level falls in CB due to an impuritydriven state that lies in the energy-gap region along $\overline{\mathrm{JKJ}^{\prime}}$ and partly along the $\overline{\mathrm{K}} \overline{\mathrm{s}}$ segment. The fully occupied quasi-1D defect state just below $1 \mathrm{eV}$ disperses in accordance with the spatial alignment of Au-O coordination. Therefore, its maximum at $\mathbf{J}^{\prime}$ for $\mathrm{c}-2 \times 2$ and at $\mathbf{J}$ for $\mathrm{d}-2 \times 2$ and $\mathrm{e}-2 \times 2$ (doubly) alternates with $\mathrm{Au}-\mathrm{O}$ bond orientation. Lower lying nearly flat state in case (d) is primarily due to $\mathrm{O} 2$ oxygens at the surface. For the cases (c) and especially (e), the stack of bands just above VBM are very similar to those of the clean surface [see Fig. 2(a) in Ref. 18] because the region of disturbance due to the interstitial $\mathrm{Au}$ is away from the first $\mathrm{TiO}_{2}$ layer for these cases and for cases with deeper interstitials.

\section{BADER ANALYSIS}

We analyzed the electronic charge density using the atom in molecule (AIM) theory with a grid-based algorithm. ${ }^{30}$ Computational Bader charge results for near-surface $\mathrm{Ti}$ and $\mathrm{O}$ atoms, and also for the Au impurity, are presented in Table II for the combined systems as well as for the clean surface. For $\mathrm{Au}-\mathrm{TiO}_{2}(001)$ structures, atomic charge states are provided for $\mathrm{O}$ and $\mathrm{Ti}$ ions that are closest to the gold site in order to better describe the local disturbance of the electronic density posed by the impurity.

The nonionic nature of Ti-O bonding in $\mathrm{TiO}_{2}$ stoichiometry, due to below-nominal charge states of $\mathrm{Ti}$ cations and $\mathrm{O}$ anions, has been previously shown. ${ }^{29,31}$ Bulk ions accumulate $Q_{\mathrm{O}}=-1.33 e$ and $Q_{\mathrm{Ti}}=+2.66 e$ Bader charges while these values are slightly lower for the corresponding surface layer species, being $Q_{\mathrm{O} 1}=-1.26 e$ and $Q_{\mathrm{Ti} 1}=+2.61 e$, due to bulk termination.

Bader charge results presented in Table II for the impurity neighboring ions demonstrate the local disturbance of the electronic density. The fluctuation in the charge density around the cation sites remains minimal upon Au incorporation. For interstitial and adsorptional cases, gold seems to be weakly bound to the lattice because of a limited charge transfer from $\mathrm{Au}$ to nearby oxygens. We obtained relatively smaller deviations in the charge states of deeper lying atoms implying a weaker contribution to surface electronic properties. When gold is substituted for Ti cations, it induces deviation in the electronic density also around the second nearest-neighbor oxygens owing to its spatially wider spread wave function. For instance, Au-Ti1 substitution in s1-1 $\times 1$ charges the third-layer oxygen to $Q_{O 3}=-1.18 e$ which is smaller than its reference value of $Q_{O 3}=-1.33 e$ at the clean surface.

In comparison with their reference values at the clean surface, a lower amount of charge accumulation at the anions around the impurity site has been obtained for all Au- $\mathrm{TiO}_{2}(001)$ structures in Table II. This elicits a weak polarization in the covalency between gold and oxygen at this surface. The Au-O and Ti-O interaction strengths can be compared based on the charge transfers in between. The electron depletion from $\mathrm{Ti}$ to $\mathrm{O}$ is clearly much larger than that from $\mathrm{Au}$ to $\mathrm{O}$ suggesting that Ti-O bond is stronger.

\section{Au dimer on $2 \times 2$}

The weakness of Au-O bond polarization in and on $\mathrm{TiO}_{2}$ surface compared to that of Ti-O opens the possibility of an 

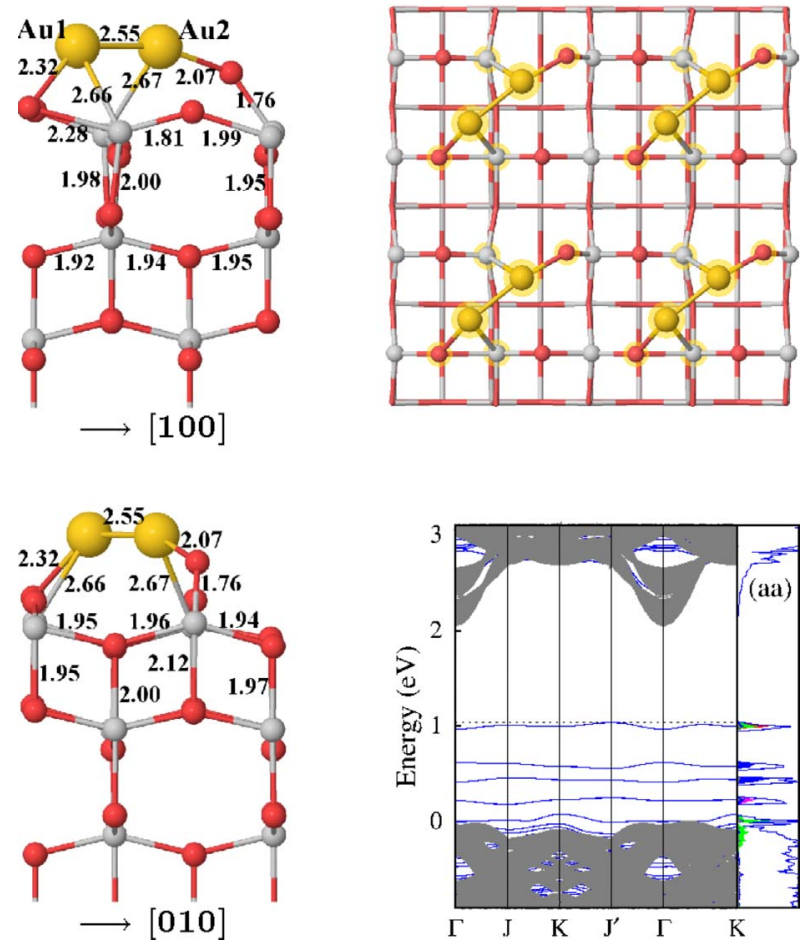

FIG. 3. (Color online) $\mathrm{Au}$ dimer on anatase $\mathrm{TiO}_{2}(001)-2 \times 2$ surface $($ aa- $2 \times 2)$. Side views of the impurity embedded slab model are shown in the left panel where all distances are given in $\AA$. Top view of gold dimers on this surface as well as the corresponding electronic structure are presented in the right panel.

important interaction between two adjacent $\mathrm{Au}$ atoms. Clearly, Au-driven impurity bands disperse relatively strongly when they are incorporated to $1 \times 1$ surface unit cell in Fig. 1. Therefore, we considered all possible adsorption configurations of $\mathrm{Au}$ dimer on $2 \times 2$ surface in order to investigate the effect of $\mathrm{Au}-\mathrm{Au}$ interaction on the electronic structure of the combined system. Figure 3 shows the minimum-energy $\mathrm{Au}$ dimer structure on the anatase $\mathrm{TiO}_{2}(001)-2 \times 2$ surface. This is obtained from an initial configuration in which one $\mathrm{Au}$ atom is placed over the Ti1-O1 bond in bridge position and the other one is located over the next Ti1-O1 bond on the back Ti-O-Ti row. They are attracted to each other due to $\mathrm{Au}-\mathrm{Au}$ interaction. Relatively speaking, with respect to the structure shown in the left top of Fig. 3, as they come closer reducing the dimer length to $2.55 \AA$, the one at the back row (Au2) pulls the nearestneighbor $\mathrm{O} 1$ off its lattice position up by $0.91 \AA$. However, the $\mathrm{O} 1$ in interaction with the Aul at the first row is elevated by only $0.06 \AA$. Surprisingly, any geometry that the surface oxygens bonded symmetrically to the dimer is energetically unfavorable.

We also examined the possibility of a dissociative adsorption for various configurations which corresponds to $0.5 \mathrm{ML}$ coverage on this surface. Our results suggest that such a dimer dissociation on the anatase surface is energetically not preferable signifying the strength of $\mathrm{Au}-\mathrm{Au}$ attraction compared to $\mathrm{Au}-\mathrm{O}$ and $\mathrm{Au}-\mathrm{Ti}$ interactions.

Electronically, gold dimer adsorption at $2 \times 2$ unit cell drives the anatase (001) surface into a semiconducting state

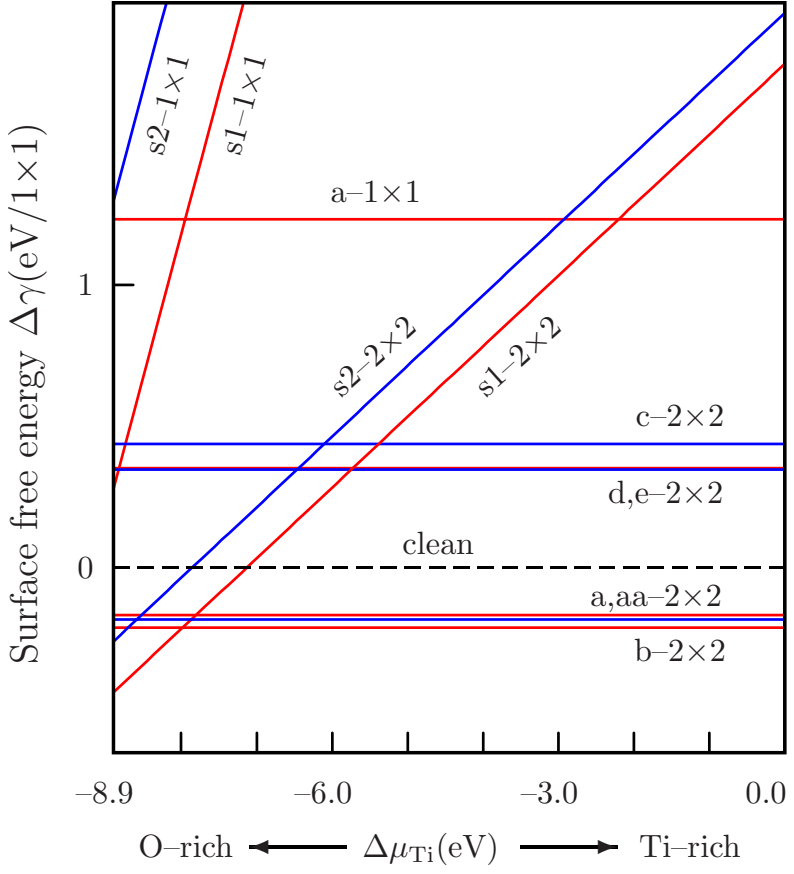

FIG. 4. (Color online) Normalized relative surface Gibbs free energies of $\mathrm{Au}-\mathrm{TiO}_{2}(001)-1 \times 1$ and $\mathrm{Au}-\mathrm{TiO}_{2}(001)-2 \times 2$ structures as a function of and over the allowed range of Ti chemical potential. $\mathrm{Au}$ impurity ions are chosen to be in thermodynamic equilibrium with ccp Au bulk phase.

such that it reduces the band gap by $1.04 \mathrm{eV}$ relative to anatase bulk VBM. The impurity band just below the Fermi energy has a minimum at $\Gamma$ and a maximum at $\mathrm{J}^{\prime}$ which gives rise to an indirect gap. This otherwise almost flat going state lies higher in energy from a group of five surfacelike bands with a separation of $0.34 \mathrm{eV}$ at $\Gamma$. It is mainly characterized by $\mathrm{Au}-\mathrm{Au}$ and $\mathrm{Au} 1-\mathrm{O} 1$ interactions with a partial mixing from the unsaturated surface oxygens. The second defect states disperses conjugately to the first one having a maximum at $\Gamma$ and a minimum at $\mathrm{J}^{\prime}$. The surface oxygen that is bonded to Au2 mainly contributes to the second and the third defect states. The next impurity band that has a minimum at $\mathbf{J}$ and a maximum at $\mathrm{J}^{\prime}$ originates from $\mathrm{Au} 2-\mathrm{O} 1$ bonding. The LDOS of unsaturated $\mathrm{O} 1$ ions extend over the $\mathrm{VB}$, also giving partial contribution to all of the defect states.

\section{THERMODYNAMIC STABILITY}

We analyzed the thermodynamic stability of goldincorporated $\mathrm{TiO}_{2}$ systems, considered in this work, assuming $\mathrm{Au}$ and $\mathrm{Ti}$ exchange between the substrate and the surrounding gas phase to account for the formation of substitutional cases. We assume experimental conditions so that $\mathrm{Au}$ is chosen to be in equilibrium with its metallic bulk phase and redefine the zero point of energy at the maximal value of $\mu_{\mathrm{Ti}}$ by introducing $\Delta \mu_{\mathrm{Ti}}=\mu_{\mathrm{Ti}}-\mu_{\mathrm{Ti}}^{\text {bulk }}$. The relative surface GFEs, $\Delta \gamma$ (or the formation energies) are plotted as a function of $\Delta \mu_{\mathrm{Ti}}$ in Fig. 4 over its allowed range of values for 12 surface structures considered in this study. The lower limit for $\mathrm{Ti}$ chemical potential has been obtained to be 
$-8.90 \mathrm{eV}$ due to the fact that the surface must keep in thermal equilibrium with the bulk anatase $\mathrm{TiO}_{2}$ phase.

Our prescription of stability of the phases can predict the structural reconstructions rather than thermally stimulated formations due to the approximation made by omitting the entropy terms in the surface GFE. The results presented in Fig. 4 suggest that $\mathrm{Au}$ incorporation at $1 \times 1$ surface is not favorable. For instance, Au substitution for the second-layer Ti at $1 \times 1$ anatase $(001)$ surface $(s 2-1 \times 1)$ is energetically the least preferable phase among the structures considered. Similarly, s1-1 $\times 1$ case is also thermodynamically unstable. Their formation energies further increase linearly toward Tirich experimental conditions. Au adsorption for 1 ML coverage would require an energy of $1.23 \mathrm{eV} / 1 \times 1$ relative to that of the clean surface.

$\mathrm{Au}$ interstitials in the $2 \times 2$ surface appears to be unfavorable compared to bulk-terminated bare anatase $\mathrm{TiO}_{2}(001)$ by the formation energies of $0.44,0.35$, and $0.35 \mathrm{eV} / 1 \times 1$ for (c), (d), and (e) cases, respectively. Surprisingly, Au implantation into the interstitial cavities deeper than the fourth atomic layer is slightly more preferable at this surface. These calculated formation energies for $\mathrm{Au}$ interstitials in anatase surface are in good agreement with the experimental results of Perkas et al. ${ }^{13}$ In an attempt to explain Au-induced crystallization of anatase through gold insertion into $\mathrm{TiO}_{2}$ which requires thermal treatment at about $80{ }^{\circ} \mathrm{C}$ by sonication, they stated that Au diffuses into the support layers and forms an intermixed layer through a multiphase diffusion across the gold support interface.

Substitutional gold impurities tend to be linearly more unstable toward Ti-rich conditions. It is also reasonable to see that $\mathrm{Au}$ substitution for a second $\mathrm{Ti}$ layer cation is energetically more unstable than that for the surface-layer Ti over the full range of allowed $\mu_{\mathrm{Ti}}$ values. On the other hand, $\mathrm{Au}(\mathrm{Ti})$ substitution has been expected to be more preferable in a Ti-poor environment. Indeed, both of the substitutional cases become more stable than the clean relaxed surface under O-rich conditions when the substrate establishes thermodynamic equilibrium with the surrounding oxygen gas phase. Figure 4 shows that such a surface structure can be realized under the limits, $\Delta \mu_{\mathrm{Ti}}<-7.84 \mathrm{eV}$ and $\Delta \mu_{\mathrm{Ti}}<-7.14 \mathrm{eV}$ for s2 and s1 cases, respectively. Furthermore, $\mathrm{Au}(\mathrm{Ti})$ substitution for the surface $\mathrm{Ti}$ atom proves to be the most stable phase for $-8.90<\Delta \mu_{\mathrm{Ti}}<-7.98 \mathrm{eV}$ that corresponds to a $\mathrm{Ti}$ poor environment.

$1 \mathrm{ML}$ gold adsorption is not preferable with a formation energy of $1.23 \mathrm{eV}$ relative to that of the clean surface. On the other hand, Au adsorbates at $2 \times 2$ unit cell as well as the dimer structure in Fig. 3 happen to be even more stable than the formation of bulk-terminated bare (001) surface which is known to be under tensile stress ${ }^{32}$ whereby $\mathrm{Au}$ bonding causes a lowering of the surface energy by releasing the surface $\operatorname{strain}^{33}$ We calculated the corresponding relative surface GFEs as $-0.17,-0.21$, and $-0.18 \mathrm{eV} / 1 \times 1$ for $\mathrm{a}-2 \times 2$, b- $2 \times 2$, and aa- $2 \times 2$ structures, respectively. Therefore, highly symmetrical structure in Fig. 2(b) appears to be the most stable phase over a wide range of experimental situations within $-7.84<\Delta \mu_{\mathrm{Ti}}<0$ from Ti-low to Ti-rich equilibrium conditions. However, the differences between the computed GFE values of these three adsorption modes are, surprisingly, not significantly large and can be accepted within the limits of a computational accuracy. In this sense, one cannot conclude that $\mathrm{a}-2 \times 2$ and aa- $2 \times 2$ are considerably less stable than $b-2 \times 2$ is. The results rather suggest that these three phases might coexist in an experimental situation where, interestingly, single $\mathrm{Au}$ atom adsorption is metallic while dimer structure is semiconducting. Therefore, overall behavior is expected to be conducting in nature.

\section{CONCLUSIONS}

In conclusion, we studied the role of Au-mediated lattice relaxations as well as the effect of the impurity itself on the electronic structure of anatase $\mathrm{TiO}_{2}(001)$ for $1 \times 1$ and 2 $\times 2$ surface unit cells. We, additionally, considered golddimer adsorption on $\mathrm{TiO}_{2}(001)-2 \times 2$ to account for the strength of $\mathrm{Au}-\mathrm{Au}$ interaction over $\mathrm{Au}-\mathrm{O}$ attraction.

In comparison with $\mathrm{Ti}$, due to its spatially wider spread wave function, gold maintains a relatively larger distance to the oxygens with which it interacts. In addition, Au exhibits an ample coordination with the neighboring oxygens and, therefore, disturbs local lattice structure. Electronically, an $\mathrm{Au}$ adsorbate transfers a limited amount of charge to the nearest-neighbor oxygens that reduces the ionicity leading to a relatively weaker impurity-oxygen interaction. On the other hand, Au can attain higher valence charge states in the case of interstitial and substitutional incorporations due to increased coordination number with the neighboring oxygens. For 1 ML impurity concentration, gold interstitial enforced local distortion causes significant reconstruction in the form of interlayer segregation due to shortened $\mathrm{Au}-\mathrm{Au}$ interaction distance. These impurity-mediated disturbances derive new defect states and strongly disperse the existing surface bands.

Single Au impurity brings a half-filled impurity state into the band gap of $\mathrm{TiO}_{2}(001)$ which pins the Fermi level leading to metallization, in addition to other filled surface and impurity-driven bands within the gap. In the adsorptional and interstitial cases, this state is derived from the CB. For Au interstitials as well as for b- $2 \times 2$, Fermi energy falls inside the CB. However, substitutional Au makes it up close to the VB also deriving empty impurity states higher in energies toward the $\mathrm{CB}$. The dispersion of the defect states depend on the impurity concentration and the Au-O interaction strength. Therefore, $1 \times 1$ cases exhibit the strongest dispersions and the adsorptional $\mathrm{Au}$ incorporations at $2 \times 2$ surface lead to flatlike defect states which represent the weakest Au-O interactions.

All Au-incorporated $\mathrm{TiO}_{2}(001)$ structures at $1 \times 1$ surface unit cells are unstable. Under strongly oxidizing, Ti-poor conditions gold tends to substitute surface Ti ions at $0.25 \mathrm{ML}$ coverage. On the other hand, for a wide range of experimental situations, from moderate to strongly reducing conditions, $\mathrm{Au}$ adsorption is thermodynamically more stable at the $2 \times 2$ support surface. Single Au adsorbates at this coverage 
bring out new acceptor sites as they reduce the surface leading to metallization. Therefore, an additional gold adsorption prefers the dimer structure rather than being dissociative. Gold dimer supported by the slab at $2 \times 2$ surface is almost as stable as the single $\mathrm{Au}$ adsorption signifying the strength and the role of $\mathrm{Au}-\mathrm{Au}$ interaction which drives the system into semiconducting regime. Consequently, in an experimental situation which realizes the coexistence of these two phases, the overall behavior is expected to be conducting.

\section{ACKNOWLEDGMENTS}

E.M. and Ş.E. acknowledge financial support from TÜBİTAK, The Scientific and Technological Research Council of Turkey (Grant No. TBAG 107T560). In conjunction with this project, computational resources were provided by ULAKBIM, Turkish Academic Network and Information Center. O.G. acknowledges the support of Turkish Academy of Sciences, TÜBA.
*Corresponding author. emete@balikesir.edu.tr

${ }^{1}$ S. Kim, S.-J. Hwang, and W. Choi, J. Phys. Chem. B 109, 24260 (2005).

${ }^{2}$ E. Kowalska, H. Remita, C. Colbeau-Justin, J. Hupka, and J. Belloni, J. Phys. Chem. 112, 1124 (2008).

${ }^{3}$ K. Ko, Y. Lee, and J. Jung, J. Colloid Interface Sci. 283, 482 (2005).

${ }^{4}$ M. Kitano, M. Takeuchi, M. Matsuoka, J. M. Thomas, and M. Anpo, Catal. Today 120, 133 (2007).

${ }^{5}$ Y. Wang and D. J. Doren, Solid State Commun. 136, 186 (2005).

${ }^{6}$ E. W. McFarland and J. Tang, Nature (London) 421, 616 (2003).

${ }^{7}$ I. Marri and S. Ossicini, Solid State Commun. 147, 205 (2008).

${ }^{8}$ M. S. Chen and W. Goodman, Science 306, 252 (2004).

${ }^{9}$ J. M. Jung, M. Wang, E. J. Kim, and S. H. Hahn, Vacuum 82, 827 (2008).

${ }^{10}$ W. Yan, B. Chen, S. M. Mahurin, V. Schwartz, D. R. Mullins, A. R. Lupini, S. J. Pennycook, S. Dai, and S. H. Overbury, J. Phys. Chem. B 109, 10676 (2005).

${ }^{11}$ J. Li and H. C. Zeng, Chem. Mater. 18, 4270 (2006).

${ }^{12}$ A. Grirrane, A. Corma, and H. Garcia, Science 322, 1661 (2008).

${ }^{13}$ N. Perkas, V. G. Pol, S. V. Pol, and A. Gedanken, Cryst. Growth Des. 6, 293 (2006).

${ }^{14}$ R. Hengerer, B. Bolliger, M. Erbudak, and M. Grätzel, Surf. Sci. 460, 162 (2000).

${ }^{15}$ A. Bouzoubaa, A. Markovits, M. Calatayud, and C. Minot, Surf. Sci. 583, 107 (2005).

${ }^{16}$ A. G. Thomas, W. R. Flavell, A. K. Mallick, A. R. Kumarasinghe, D. Tsoutsou, N. Khan, C. Chatwin, S. Rayner, G. C.
Smith, R. L. Stockbauer, S. Warren, T. K. Johal, S. Patel, D. Holland, A. Taleb, and F. Wiame, Phys. Rev. B 75, 035105 (2007).

${ }^{17}$ S. Munnix and M. Schmeits, Phys. Rev. B 30, 2202 (1984).

${ }^{18}$ A. Beltrán, J. R. Sambrano, M. Calatayud, F. R. Sensato, and J. Andrés, Surf. Sci. 490, 116 (2001).

${ }^{19}$ M. Lazzeri, A. Vittadini, and A. Selloni, Phys. Rev. B 63, 155409 (2001).

${ }^{20}$ M. Calatayud and C. Minot, Surf. Sci. 552, 169 (2004).

${ }^{21}$ J. P. Perdew, K. Burke, and M. Ernzerhof, Phys. Rev. Lett. 77, 3865 (1996).

${ }^{22}$ P. E. Blöchl, Phys. Rev. B 50, 17953 (1994).

${ }^{23}$ G. Kresse and D. Joubert, Phys. Rev. B 59, 1758 (1999).

${ }^{24}$ G. Kresse and J. Hafner, Phys. Rev. B 47, 558 (1993).

${ }^{25}$ G.-X. Qian, R. M. Martin, and D. J. Chadi, Phys. Rev. B 38, 7649 (1988).

${ }^{26}$ K. Reuter and M. Scheffler, Phys. Rev. B 65, 035406 (2001).

${ }^{27}$ B. Meyer, Phys. Rev. B 69, 045416 (2004).

${ }^{28}$ V. Timon, S. Brand, S. J. Clark, M. C. Gibson, and R. A. Abraham, Phys. Rev. B 72, 035327 (2005).

${ }^{29}$ E. Mete, D. Uner, O. Gülseren, and Ş. Ellialtığlu, Phys. Rev. B 79, 125418 (2009).

${ }^{30}$ E. Sanville, S. D. Kenny, R. Smith, and G. Henkelman, J. Comput. Chem. 28, 899 (2007).

${ }^{31}$ M. Calatayud, P. Mori-Sánchez, A. Beltrán, A. M. Pendás, E. Francisco, J. Andrés, and J. M. Recio, Phys. Rev. B 64, 184113 (2001).

${ }^{32}$ M. Lazzeri and A. Selloni, Phys. Rev. Lett. 87, 266105 (2001).

${ }^{33}$ A. Vittadini and A. Selloni, J. Chem. Phys. 117, 353 (2002). 